\section{Sandwich Bone Augmentation (SBA) in immediate implant placement post-dentoalveolar trauma: a case report}

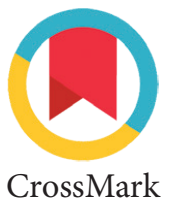

\author{
Anton* , Poerwati S. Rahajoe, Bambang Dwirahardjo
}

\title{
Abstract
}

Objective: Discussing the merits/demerits of the application of SBA procedure with titanium mesh as an alternative solution for immediate implant placement in the socket with dentoalveolar trauma-induced buccal bone defect.

Methods: An 18-year-old female patient visited our department, presenting with a history dentoalveolar trauma and a loss of tooth 21. Clinical examination during the implant placement procedure exposed a socket with buccal bone defect. SBA with autogenous chin bone graft combined with DFDBA allograft and stabilized with titanium mesh (Ti-Mesh) for buccal defect on which flap reposition was done with tension-free primary closure.
Results: Ti-Mesh was removed after 3 months, our follow-up examinations revealed the following: there was no sign of inflamation, implant was in stable condition, and new bone formation was developing. Subsequently, healing abutment was placed. A one-year observation suggested a good clinical retention with no luxation observed, along with decent functional and aesthetic results. CBCT evaluation showed buccal bone thickness was preserved.

Conclusion: Sandwich bone augmentation with stabilized titanium mesh provides satisfying results in treating horizontal buccal bone defect.
Department of Oral and Maxillofacial Surgery, Faculty of Dentistry, Universitas Gadjah Mada, Jogjakarta, Indonesia

*Corresponding to: Anton, Department of Oral and Maxillofacial Surgery, Faculty of Dentistry, Universitas Gadjah Mada, Jogjakarta, Indonesia antonleebm@mail.ugm.ac.id

Received: 9 November 2017 Revised: 14 November 2017 Accepted: 15 November 2017 Available online: 1 december 2017

Keywords: Dentoalveolar trauma, Horizontal defect, Sandwich bone augmentation, Titanium mesh Cite this Article: Anton, Rahajoe PS, Dwirahardjo B. 2017. Sandwich Bone Augmentation (SBA) in immediate implant placement postdentoalveolar trauma: a case report. Journal of Dentomaxillofacial Science 2(3): 198-201. D0I: 10.15562/jdmfs.v2i3.643

\section{Introduction}

Dentoalveolar fracture is often encountered in daily practice and is mostly caused by falling, collision, sports mishaps/accidents, or traffic accidents and usually affect the anterior part of the face. Dentoalveolar trauma can cause fracture, buccal bone loss, displacement of anterior tooth, and missing tooth; all of these can alter patients' quality of life in many ways, including, poor aesthetics, difficulty in speech and adverse psychological efects. ${ }^{1}$ Missing tooth associated with dentoalveolar trauma can cause alveolar bone resorption 1.5-2 mm vertically and 40-50\% horizontally in 6-12 months. Dentoalveolar fracture with buccal bone loss may increase difficulty in obtaining initial stability for implant placement. ${ }^{1,2}$

This article reports a case of dentoalveolar trauma with missing tooth 21 and buccal bone defect which was rehabilitated using immediate implant placement and SBA stabilized with titanium mesh.

\section{Case Report}

An 18 years old woman came to the Emergency Department after she met with a traffic accident, which resulted in collision in the anterior part of the face accompanied with tooth loss, and there was no history of head trauma. There was no history of allergy or any other systemic diseases. Physical examinations showed vital signs were normal. Excoriation was found in lip and right lip commissure. Intraoral examination showed tooth 21 avulsed with open wound and buccal bone loss; tooth 11, 12 and 23 appeared to be fractured, class I Ellis, 22 luxated figure 1. Treatment procedures carried out included injection of human ATS 1500 IU, wound management, maxillary arch bar fixation for 3 weeks, and medication administration. Immediate dental implant with SBA was planned 2 months after.

After 2-month follow-up, we found that luxation of tooth 22 was still persisting, and after flap reflection we could see three-fourth of the buccal alveolar bone was lost figure 2. As a result, extraction of tooth 22 became inevitable and two immediate dental implant placements with SBA technique were planned. Trapezium flap was made at distal line angle of tooth 11 and 22.

Implant bed preparation was done at ideal three-dimensional position, slightly to palatal, roughened surface and dental implant measuring $4 \times 13.5 \mathrm{~mm}$ was placed to obtain primary stability figure $3 \mathrm{~A}$, by decortication of buccal intra-marrow around the implant. Further steps carried out 


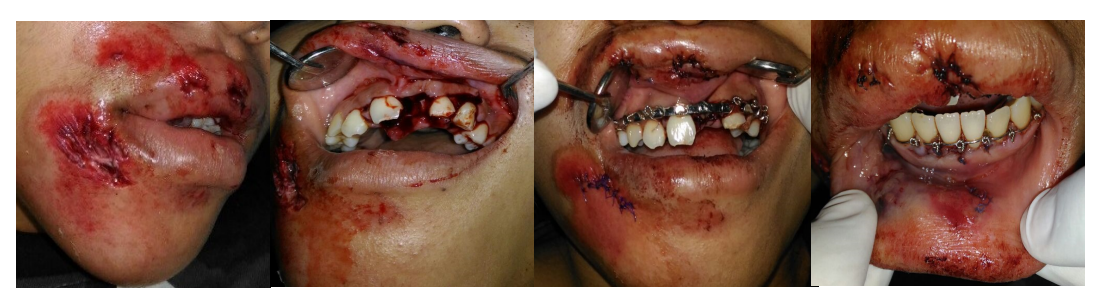

Figure 1 Extra-oral and intra-oral appearance before and after treatment

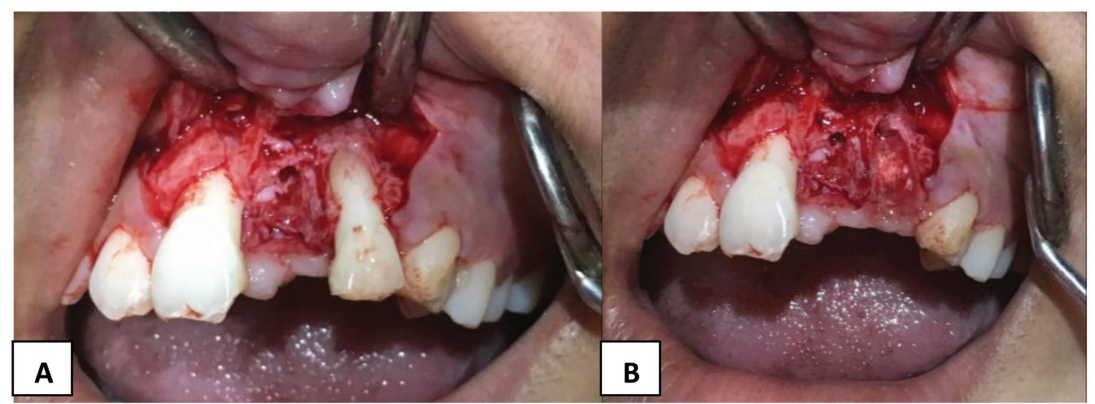

Figure 2 A. 2-month post-trauma observation-we can see the deficit of horizontal alveolar bone and third-degree luxation of tooth 22; B. Post-extraction of tooth 22

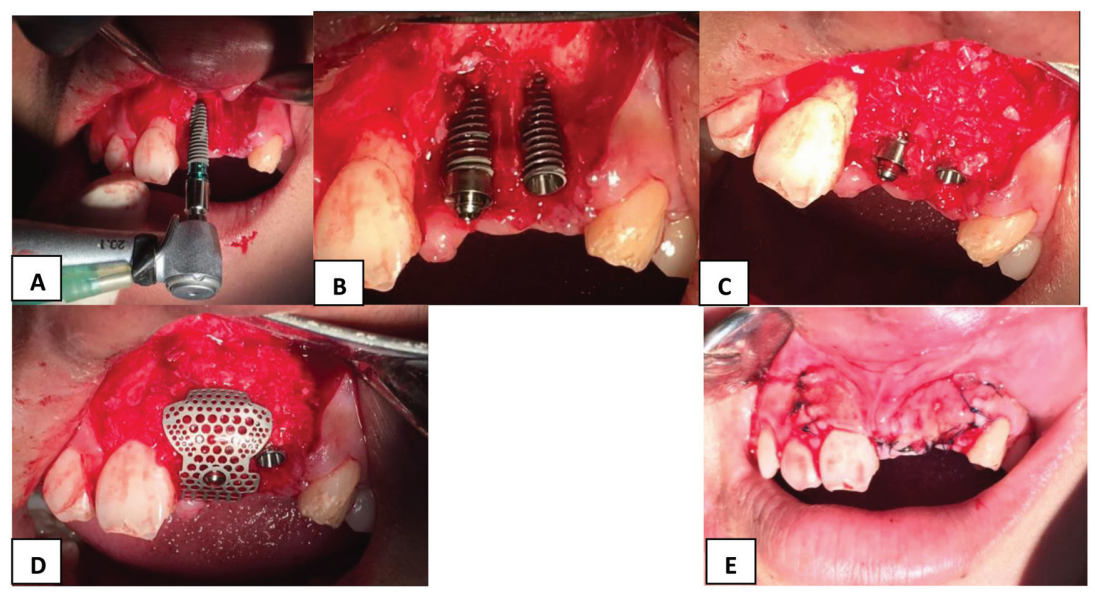

Figure 3 A. Dental implant placement at ideal position and tightened until $30 \mathrm{~N}$ torque; B-C. Exposed implant fixture covered with autograft + DFDBA; D. Ti-Mesh Instalment; E. Primary tension-free flap closure

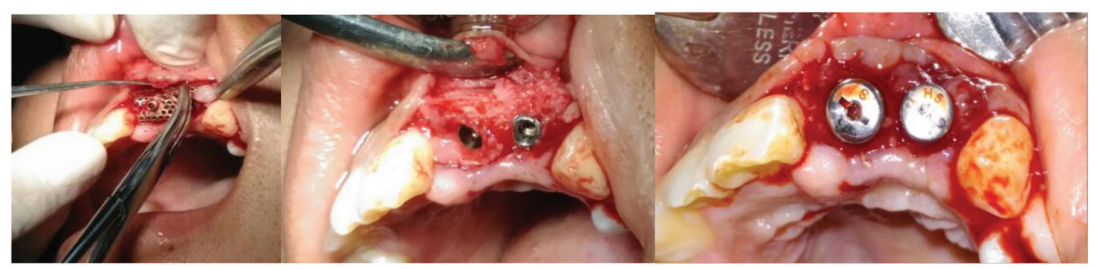

Figure 4 A. Ti-Mesh removal, B. Follow-up examination 3 months after sandwich bone augmentation revealed new bone formation around the implant fixture, C. Healing abutment insertion

included autogenous cortical cancellous bone graft harvesting from mandibular simphysis using auto bone collector drill for covering exposed implant fixture figure $3 \mathrm{~B}$ combined with demineralized

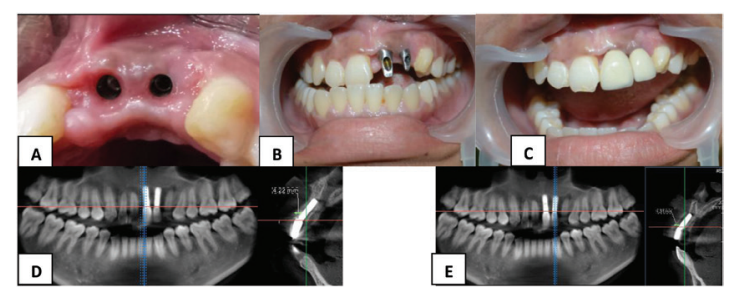

Figure 5 A. 2 months after the healing abutment insertion; B and C. Milling abutment and crown insertion; D and E. One-year follow-up CBCT evaluation showed buccal bone thickness was preserved

freeze-dried bone allograft (DFDBA) figure 3C. Bone graft stabilization and space maintenance were achieved obtained using Ti-Mesh figure 3C. Flap closure figure $3 \mathrm{E}$ was done under tension-free condition using periosteal flap-release technique, so that primary closure could be achieved and flap remained in stable condition to protect $\mathrm{Ti}^{-}$ Mesh and graft material.

Ti-Mesh was removed after 3 months, clinically resulting in total absence of inflammation. New bone formation around the implant fixture can be seen after flap reflection figure 4A and Ti-Mesh removal; all implants are in stable condition figure $4 \mathrm{~B}$. Healing abutments were inserted immediately figure $5 \mathrm{C}$ for gingival contouring and suturing was performed.

Follow-up 2 months after the healing abutment placement showed no subjective complaints, no sign of inflammation, and improved aesthetics in gingival emerging profile figure $5 \mathrm{~A}$. Impression was performed at the implant for the fabrication of crown figure 5C. A one-year observation found good clinical retention with no luxation, along with decent functional and aesthetic results. CBCT evaluation showed buccal bone thickness was preserved figure 5D-E.

\section{Discussion}

Dentoalveolar fracture can be defined as the damage or loss of hard tissue continuity including tooth and alveolar bone due to trauma or fracture, and other damages such as avulsion, subluxation, or tooth fracture. Dentoalveolar fracture can occur with or without buccal bone loss and it is usually a result of minor accidents such as falling, collision during play, sports events, or any iatrogenic cause. ${ }^{1,2}$

In the present case, dentoalveolar fracture was compounded with buccal bone loss and tooth loss, and we found that implant placement may need additional GBR procedure to augment the alveolar bone and to create sufficient foundation for precise and stable implant placement. ${ }^{3,4}$ GBR procedure is a technique that is uniquely designed to augment 
the alveolar bone in the edentulous area with future prospect of implant placements or augmentation of exposed body implant. The GBR principle inspired from GTR (guided tissue regeneration) principle postulates that specific external additional cells are required to augment inhabiting cells so that new tissue regeneration can be accelerated. Osteoblast cells with ability to promote new alveolar bone remodeling are also required for GBR. ${ }^{5,6}$

The high success rate of GBR, as much as 95\% of the cases, and its predictability in yielding good outcomes in dental implant application are indeed well known. Dental implant placement in the alveolar bone deficit area can be done directly. Clinical procedure for GBR must follow the biological principle known as the PASS principle, based on which the following conditions were assessed: in which area the primary wound closure should take place, angiogenesis, space creation/maintenance and stability of blood clot and implant fixture. SBA technique was designed following this principle in order to obtain optimal bone regeneration. ${ }^{7-9}$ The main principle of SBA technique is the application of different layers of bone graft material to mimic alveolar bone layer and then cover with membrane as a barrier. Other steps involved incorporating the following: inner layer filled with easily resorbed cancellous bone graft, outer layer composed by cortical bone graft as a scaffold to facilitate form and maintaining space and the outermost layer created using membrane barrier. ${ }^{10,11}$

In this case, SBA technique was used for its advantages such as short treatment time, ideal restoration, less morbidity, more comfortable, and lower costs. On the other hand, the main disadvantages of SBA technique is the technical difficulty associated with obtaining primary stabilization of implant inserted in the horizontal defect. ${ }^{9,11}$ This becomes an important issue because micro-movement can cause implant encapsulation that leads to implant failure. ${ }^{12,13}$ We used DFDBA as a bone graft with collagen component, which is the most important component of bone tissue and Bone Morphogenic Protein (BMP) that can trigger new bone formation in the operation area. Contact between DFDBA and the bone will create an ideal environment for osteogenic cell migration and proliferation. Intra-marrow decortication around the implant was performed using $0.5 \mathrm{~mm}$ round bur to increase regional accelerator phenomenon and angiogenesis. ${ }^{8,9}$

In this case, flap was attached to periosteal dissection to increase flap mobility and reduce tension, as this will facilitate stabilization of blood clot and protection of operation site during healing process. ${ }^{8}$ The most common complication that can occur is wound dehiscence, but in this case it did not happen and optimal bone regeneration process could be achieved. Bone volume reduction during the bone remodeling process may happen at dehiscence or at exposed operation site due to bacterial colonization, vascularization reduction in the operation site, and contamination caused by external agents. ${ }^{14}$

Membrane barrier is a material used for GBR to prevent the migration of epithelial/nonosteogenic soft-tissue-forming cells into the bone graft. Membrane material for GBR technique must qualify the following requirements: biocompatible, nontoxic, non-antigenic, and it should not cause severe inflammatory response to surrounding tissue. ${ }^{15,16}$ Non-resorbable membrane was used initially, but, in the recent years, bioresorbable or biodegradable membranes were developed for use in cases involving only small or minor defects . Resorbable membrane is available in many forms to suit various requirements. ${ }^{2,15,16}$

Polytetrafluoroethylene (PTFE) and Titanium mesh are the most commonly used non-resorbable membranes for treating large alveolar bone defects, which requires prolong space maintaining and ability to hold compression force from surrounding tissue until the remodeling process was completely done. ${ }^{2,6,16}$ In this case, titanium mesh was used because of its many advantages: it is flexible, it can be easily formed, and its porosity can facilitate direct blood supply from periosteum to tissue and bone graft below it. The main disadvantages of using non-resorbable membrane include the following: the need for second surgical procedure to remove the membrane that can decrease patient's comfort, increasing costs and causing little resorption at crestal area due to new flap opening. . $^{2,16}$

\section{Conclusion}

Implant placement in dentoalveolar fracture cases with horizontal bone defect using sandwich bone augmentation technique and titanium mesh stabilization can provide satisfying results, specifically in the management of horizontal buccal bone defects involving extensive bone defects that need immediate implant placements.

\section{Conflict of Interest}

The authors report no conflict of interest.

\section{References}

1. Turkistani J, Hanno A. Recent trends in the management of dentoalveolar traumatic injuries to primary and young permanent teeth. Dent Traumatol 2011 ;27: 46-54. 
2. Andreasen JO, Andreasen FM, Andrersson L. Textbook and color atlas of traumatic injuries to the teeth. 4th ed. Wiley-Blackwell 2007;203: 615.

3. Liu J, Kerns DG. Mechanisms of guided bone regeneration: a review. Open Dent J 2014;8: 56-65.

4. Lee A, Brown D, Wang H-L. Sandwich bone augmentation for predictable horizontal bone augmentation. Implant Dent 2009;18: 282-290.

5. Hwang D, Sonick M. Guided bone regeneration: concepts and materials. Implant Site Dev 2015;153-178.

6. Hitti R, Kerns D. guided bone regeneration in the oral cavity: a review. Open Pathol J 2011;5: 33-45.

7. $\mathrm{Fu} \mathrm{JH}, \mathrm{Oh} \mathrm{TJ}$, Benavides E, et al. A randomized clinical trial evaluating the efficacy of the sandwich bone augmentation technique in increasing buccal bone thickness during implant placement surgery: I. Clinical and radiographic parameters. Clin Oral Implants Res 2014;25: 458-467.

8. Wang HL, Boyapati L. "PASS" Principles for Predictable Bone Regeneration. Implant Dent 2006;15: 8-17.

9. Wang H-L, Misch C, Neiva RF. Sandwich bone augmentation technique: rationale and report of pilot cases. Int J Periodontics Restorative Dent 2004;24: 232-245.

10. Fu J, Wang HL. Horizontal bone augmentation: the decision tree. Int $\mathrm{J}$ Periodontics Restorative Dent 2011;31: 429-436.

11. Fu J, Wang $\mathrm{H}$. The Sandwich Bone Augmentation Technique. Clin Adv Periodontics 2012;2: 172-177.
12. Bottino MC, Thomas V, Schmidt G, et al. Recent advances in the development of GTR/GBR membranes for periodontal regeneration - A materials perspective. Dental Materials 2012;28: 703-721.

13. Nyman S, Lang NP, Buser D, et al. Bone regeneration adjacent to titanium dental implants using guided tissue regeneration: a report of two cases. Int J oral Maxillofac Implant 1990;5: 9-14.

14. Pellegrini G, Pagni G, Rasperini G. Surgical approaches based on biological objectives: GTR vs GBR techniques. Int J Dent 2013;1-13.

15. Khan $\mathrm{R}$, Khan $\mathrm{MH}$, Bey A. Use of collagen as an implantable material in the reconstructive procedures - an overview. Biology and Medicine 2011;3: 25-32.

16. Rakhmatia YD, Ayukawa Y, Furuhashi A, et al. Current barrier membranes: Titanium mesh and other membranes for guided bone regeneration in dental applications. Journal of Prosthodontic Research 2013;57: 3-14.

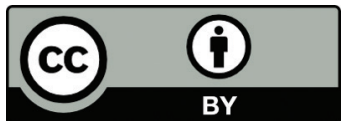

This work is licensed under a Creative Commons Attribution 